\title{
MULTILEVEL BLOCKING MONTE CARLO SIMULATIONS FOR QUANTUM DOTS
}

\author{
R. EGGER \\ Institute for Theoretical Physics, University of California, \\ Santa Barbara, CA 93106, USA \\ C.H. Mak \\ Department of Chemistry, University of Southern California, \\ Los Angeles, CA 90089-0482, USA
}

\begin{abstract}
This article provides an introduction to the ideas behind the multilevel blocking (MLB) approach to the fermion sign problem in path-integral Monte Carlo simulations, and also gives a detailed discussion of MLB results for quantum dots. MLB can turn the exponential severity of the sign problem into an algebraic one, thereby enabling numerically exact studies of otherwise inaccessible systems. Low-temperature simulation results for up to eight strongly correlated electrons in a parabolic $2 \mathrm{D}$ quantum dot are presented.
\end{abstract}

\section{Introduction: The fermion sign problem}

Quantum Monte Carlo (QMC) techniques are among the most powerful methods for the computer simulation of strongly correlated many-fermion systems, capable of delivering numerically exact results. This article deals with a finite-temperature QMC method, namely path-integral Monte Carlo (PIMC), which is based on a discretized path-integral formulation of the imaginary-time many-fermion propagator. Despite its promises, applications of QMC to many-fermion systems have been severely handicapped by the infamous 'sign problem'. 1 Exchange leads to non-positive-definite fermionic density matrix elements, and the sign cancellations arising from sampling fermion paths manifest themselves as a small signal-to-noise ratio, $\eta \sim \exp \left(-N \beta E_{0}\right)$, that vanishes exponentially with both particle number $N$ and inverse temperature $\beta=1 / k_{B} T$ ( $E_{0}$ is a system-dependent energy scale). Apart from variational or approximate treatments, such as the fixed-node approximation, a solution to the sign problem in QMC simulations had remained elusive.

In a recent paper, 3 we developed a general scheme for tackling the sign problem in PIMC simulations. The method has been applied to interacting electrons in a quantum dot 3 and to the real-time dynamics of simple few-degrees-of-freedom systems. 1 This multilevel blocking (MLB) approach is a systematic implementation of a simple blocking strategy. The theorem behind the blocking strategy asserts that by sampling groups of paths ('blocks') at the same time, the sign problem can always be reduced compared to sampling single paths as would be done normally (see Sec. 2.1 for details). By suitably bunching paths together into sufficiently small blocks, the sign cancellations among paths within the same block can be accounted for without the sign problem, simply because there is no sign problem for a sufficiently small system. The MLB approach is then able to turn the exponential severity of the sign problem into an algebraic one. In practice, that implies that significantly larger systems can be now studied by PIMC.

The purpose of this article is twofold. First, we want to present the basic ideas 
underlying MLB, omitting technical implementation issues discussed in our original work. In particular, the reason why blocking paths together is helpful will be discussed in some detail. The second aim is to demonstrate the practical usefulness of MLB for interesting quantum many-fermion applications. In Sec. 3, MLB results are presented for strongly correlated electrons in a $2 \mathrm{D}$ quantum dot.

\section{Multilevel blocking approach}

\subsection{Blocking strategy}

We consider a many-fermion system whose state is described by a set of quantum numbers $\vec{r}$ denoting, e.g., the positions and spins of all particles. These quantum numbers may correspond to electrons living on a lattice or in continuous space. For notational simplicity, we focus on calculating the equilibrium expectation value of a diagonal operator or correlation function (this can be easily generalized),

$$
\langle A\rangle=\frac{\sum_{\vec{r}} A(\vec{r}) \rho(\vec{r}, \vec{r})}{\sum_{\vec{r}} \rho(\vec{r}, \vec{r})},
$$

where $\sum_{\vec{r}}$ represents either a summation for the case of a discrete system or an integration for a continuous system. In PIMC applications, imaginary time is discretized into $P$ slices of length $\epsilon=\beta / P$. Inserting complete sets at each slice $m=1, \ldots, P$, and denoting the corresponding configuration on slice $m$ by $\vec{r}_{m}$, the diagonal elements of the reduced density matrix at $\vec{r}=\vec{r}_{P}$ entering Eq.(11) read

$$
\rho\left(\vec{r}_{P}, \vec{r}_{P}\right)=\sum_{\vec{r}_{1}, \ldots, \vec{r}_{P-1}} \prod_{m=1}^{P}\left\langle\vec{r}_{m+1}\left|e^{-\epsilon H}\right| \vec{r}_{m}\right\rangle .
$$

One can then construct accurate analytical approximations for the short-time propagator. This formulation of the problem excludes effective actions such as those arising from an integration over the fermions using the Hubbard-Stratonovich transformation, 1 , since they generally lead to long-ranged imaginary-time interactions. The MLB approach suitable for such systems is described elsewhere.

In dealing with a many-fermion system, we need to sum over all particle permutations and the best way to do this is to antisymmetrize the short-time propagators explicitly instead of letting the Monte Carlo handle it. This leads to the appearance of fermion determinants. Strictly speaking, the antisymmetrization has to be done only on one time slice, but the intrinsic sign problem is much better behaved if one antisymmetrizes on all time slices.

Choosing the absolute value of the product of the short-time propagators in Eq.(2) as the positive definite $\mathrm{MC}$ weight function $P[X]$, one then accumulates the sign $\Phi[X]$ associated with every path $X=\left(\vec{r}_{1}, \ldots, \vec{r}_{P}\right)$ sampled,

$$
\langle A\rangle=\frac{\sum_{X} P[X] \Phi[X] A[X]}{\sum_{X} P[X] \Phi[X]} .
$$

Assuming that there are no exclusivity problems in the numerator so that $A[X]$ is well-behaved, we can gauge the severity of the sign problem in terms of the variance 
of the denominator,

$$
\sigma^{2} \approx \frac{1}{N_{s}}\left(\left\langle\Phi^{2}\right\rangle-\langle\Phi\rangle^{2}\right)
$$

where $N_{s}$ is the number of $\mathrm{MC}$ samples taken and the stochastic averages are calculated with $P$ as the weight function. For the fermion sign problem, where $\Phi= \pm 1$ and hence $\left\langle\Phi^{2}\right\rangle=1$, the variance of the signal is controlled by the size of $|\langle\Phi\rangle|$.

Remarkably, one can achieve considerable progress by simply blocking paths together. 6 By this we mean instead of sampling single paths in the MC, we can sample sets of paths ("blocks"). Under such a blocking operation, the stochastic estimate for $\langle A\rangle$ takes the form

$$
\langle A\rangle=\frac{\sum_{B}\left(\sum_{X \in B} P[X] \Phi[X] A[X]\right)}{\sum_{B}\left(\sum_{X \in B} P[X] \Phi[X]\right)},
$$

where one first sums over the configurations belonging to a block $B$ in a way that is not affected by the sign problem, and then stochastically sums over the blocks. The summation within a block must therefore be done non-stochastically, or alternatively the block size must be chosen sufficiently small. Of course, there is considerable freedom in how to choose this blocking.

Let us analyze the variance $\sigma^{\prime 2}$ of the denominator of Eq.(5). We first define new sampling functions in terms of the blocks which are then sampled stochastically,

$$
P^{\prime}[B]=\left|\sum_{X \in B} P[X] \Phi[X]\right|, \quad \Phi^{\prime}[B]=\operatorname{sgn}\left(\sum_{X \in B} P[X] \Phi[X]\right) .
$$

Rewriting the average sign in the new representation, i.e., using $P^{\prime}[B]$ as the weight, then inserting the definition of $P^{\prime}$ and $\Phi^{\prime}$ in the numerator,

$$
\left\langle\Phi^{\prime}[B]\right\rangle=\frac{\sum_{B} P^{\prime}[B] \Phi^{\prime}[B]}{\sum_{B} P^{\prime}[B]}=\frac{\sum_{X} P[X] \Phi[X]}{\sum_{B} P^{\prime}[B]},
$$

and comparing to the average sign in the standard representation using $P[X]$ as the weight, we obtain

$$
\frac{\left|\left\langle\Phi^{\prime}\right\rangle\right|}{|\langle\Phi\rangle|}=\frac{\sum_{X} P[X]}{\sum_{B} P^{\prime}[B]}
$$

By virtue of the Schwarz inequality,

$$
\sum_{B} P^{\prime}[B]=\sum_{B}\left|\sum_{X \in B} P[X] \Phi[X]\right| \leq \sum_{B}\left|\sum_{X \in B} P[X]\right|=\sum_{X} P[X],
$$

we see that for any kind of blocking, the average sign improves (or stays the same), $\left|\left\langle\Phi^{\prime}\right\rangle\right| \geq|\langle\Phi\rangle|$. Furthermore, since $\left\langle\Phi^{\prime 2}\right\rangle=\left\langle\Phi^{2}\right\rangle=1$, we conclude from Eq.(伭) that

$$
\sigma^{\prime 2} \leq \sigma^{2}
$$

and hence the signal-to-noise ratio is always improved upon blocking configurations together. Clearly, the worst blocking one could possibly choose would be to group 
the configurations into two blocks, one with positive sign and the other with negative sign. In this case, blocking yields no improvement whatsoever, and the ' $\leq$ ' becomes '=' in Eq.(7). It is apparent from Eq.(7) that the blocking strategy provides a systematic approach to reduce the sign problem.

\subsection{Multilevel blocking approach}

A direct implementation of the strategy described in Sec. 2.1 does indeed improve the sign problem but will not remove its exponential severity. The reason is simply that for a sufficiently large system, there will be too many blocks, and once the signals coming from these blocks are allowed to interfere, one again runs into the sign problem (albeit with a smaller scale $E_{0}$ ). The resolution to this problem comes from the multilevel blocking (MLB) approach 1 where one applies the blocking strategy in a recursive manner to the blocks again. In a sense, we form new blocks containing a sufficiently small number of elementary ones, and repeat this process until only one block is left.

Instead of allowing for an uncontrolled interference of the block signals, we therefore subdivide the block space by forming a hierarchy of 'levels' $\ell=0, \ldots, L$, where the Trotter number must be of the form $P=2^{L}$. All elementary blocks are distributed onto these levels, and a MC sweep proceeds from the bottom level $\ell=0$ up to the top level $\ell=L$. When sampling on a given level $\ell<L$, no sign problem is present if sufficiently small block sizes have been chosen. The nontrivial computational task consists of finding a controllable way of transferring interference information from level $\ell$ to $\ell+1$. It is then indeed possible to proceed without numerical instabilities from the bottom up to the top level, where the expectation values of interest are computed.

The blocks at the top level still have different signs, but the interference is however much weaker than in the original formulation. Empirically, we found that the exponential severity is turned into an only algebraic one. The algebraic scaling can also be derived by a simple argument. 5 For lack of space, we refer the interested reader to our original paper 2 for practical implementation issues of the MLB algorithm, and now turn to an application.

\section{Application: Quantum dots}

Quantum dots are solid-state artificial atoms with tunable properties. Confining a small number of electrons $N$ in a 2D electron gas in semiconductor heterostructures, novel effects due to the interplay between confinement and the Coulomb interaction have been observed experimentally. For small $N$, comparison of experiments to the generalized Kohn theorem indicates that the confinement potential is parabolic and hence quite shallow compared to conventional atoms. Employing the standard electron gas parameter $r_{s}$ to quantify the correlation strength, a Fermi-liquid-like picture with the sequential filling of single-particle orbitals is applicable only for small $r_{s}$. In the low-density (strong-interaction) limit of large $r_{s}$, however, classical considerations suggest a Wigner crystal-like phase with electrons spatially arranged in shells, 6 termed Wigner molecule due to its finite extent. We are particularly 
interested in the crossover regime between these two limits, where single-particle or classical descriptions break down, and basically no other sufficiently accurate method is available. Exact diagonalization is limited to very small $N$ since one otherwise introduces a huge error due to the truncation of the Hilbert space. HartreeFock (and related) calculations become unreliable for large $r_{s}$ and incorrectly favor spin-polarized states. Furthermore, density functional calculations can introduce uncontrolled approximations. In fact, by comparing with our numerically exact data, symmetry-broken spin density solutions found in a recent density functional study, 9 and later in a Hartree-Fock calculation, 10 are most likely artefacts of the approximations involved. A variatipnal Monte Carlo with a fixed-node approximation has been employed by Bolton. 11 Comparing this to our exact calculations, we find that typical fixed-node errors in the total energy for $N>5$ are of the order of $10 \%$. It is then clear that one must resort to exact methods, especially when examining spin-dependent quantities.

\subsection{Model}

We study a clean $2 \mathrm{D}$ parabolic quantum dot with zero magnetic field,

$$
H=\sum_{j=1}^{N}\left(\frac{\vec{p}_{j}^{2}}{2 m^{*}}+\frac{m^{*} \omega_{0}^{2}}{2} \vec{x}_{j}^{2}\right)+\sum_{i<j=1}^{N} \frac{e^{2}}{\kappa\left|\vec{x}_{i}-\vec{x}_{j}\right|}
$$

Here the electron positions (momenta) are given by $\vec{x}_{j}\left(\vec{p}_{j}\right)$, their effective mass is $m^{*}$, and the dielectric constant is $\kappa$. The MLB calculations are carried out at fixed $N$ and fixed $z$-component of the total spin, $S=\left(N_{\uparrow}-N_{\downarrow}\right) / 2$. We have studied the energy, $E=\langle H\rangle$, the radial charge and spin densities $\rho(r)$ and $s_{z}(r)$ normalized to $\int_{0}^{\infty} d r 2 \pi r \rho(r)=N$ and $\int d r 2 \pi r s_{z}(r)=S$, and the two-particle correlation function

$$
g_{S}(\vec{x})=\frac{2 \pi l_{0}^{2}}{N(N-1)}\left\langle\sum_{i \neq j=1}^{N} \delta\left(\vec{x}-\vec{x}_{i}+\vec{x}_{j}\right)\right\rangle .
$$

$g_{S}$ is isotropic, and with $y=r / l_{0}$ prefactors are chosen such that $\int_{0}^{\infty} d y y g_{S}(y)=1$. The confinement length scale $l_{0}=\sqrt{\hbar / m^{*} \omega_{0}}$ allows the interaction strength to be parametrized by $\lambda=l_{0} / a=e^{2} / \kappa \omega_{0} l_{0}$, where $a$ is the effective Bohr radius of the artificial atom. For any given $N$ and $\lambda$, the parameter $r_{s}=r^{*} / a$ follows by identifying $r^{*}$ with the first maximum in $\sum_{S} g_{S}(r)$. In the simulations, unless noted otherwise, the temperature was set to $k_{B} T=0.1 \hbar \omega_{0}$. Data were collected from several $10^{4}$ samples for each parameter set $\{N, S, \lambda\}$, with a typical CPU time requirement of a few days (for each set) on a SGI Octane workstation. To check our code, we have accurately reproduced the known exact solution for $N=2$.

\subsection{Charge and spin densities}

Figure 1 shows the radial charge density $\rho(r)$ of the spin-polarized state $S=N / 2$ for $N=5$ to 8 electrons. For $\lambda=2$, increasing $N$ does not change $\rho(r)$ qualitatively, but the situation is different for strong interactions $(\lambda=8)$, where one can observe 

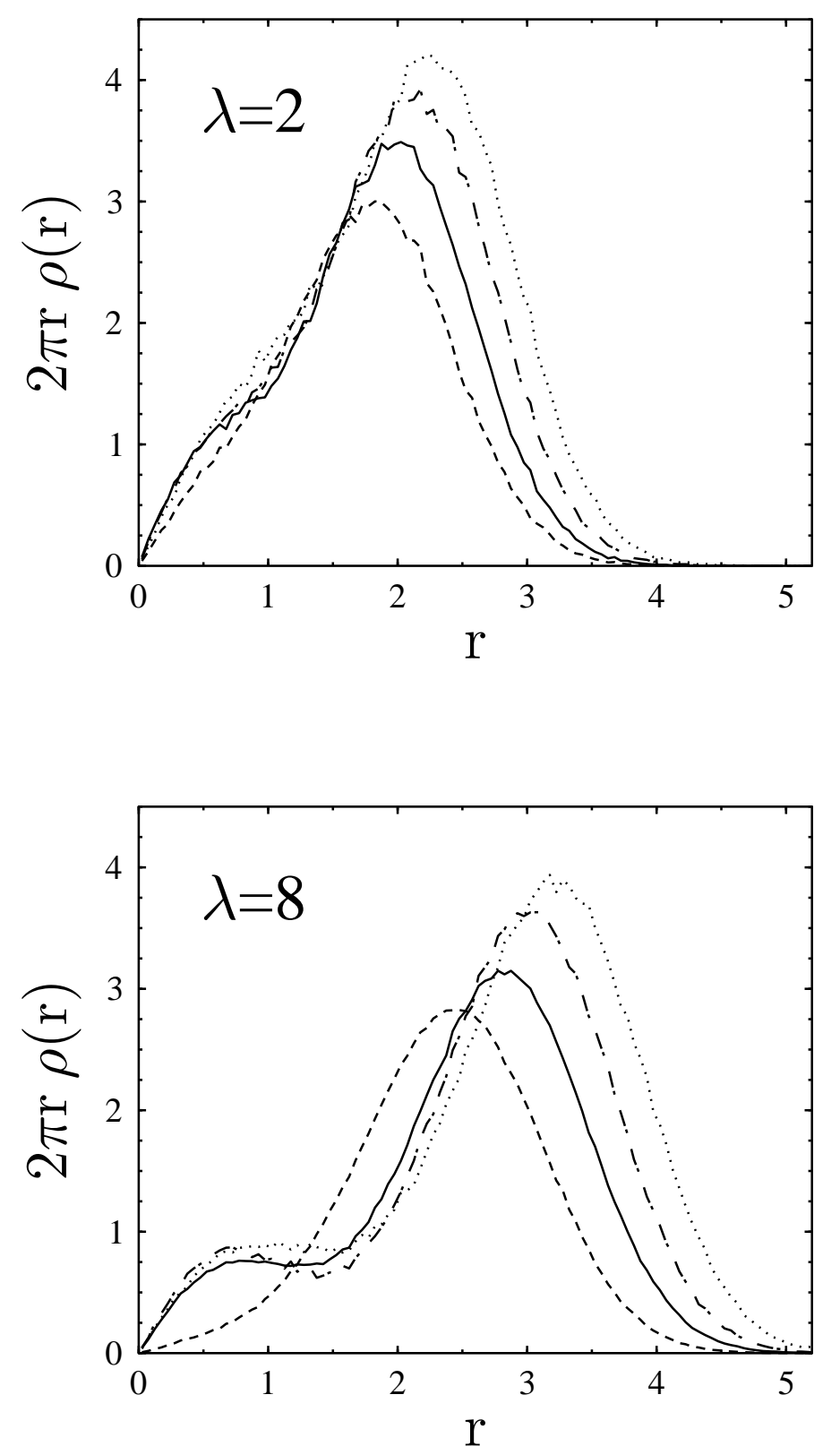

Figure 1: Density $\rho(r)$ of the spin-polarized state $(S=N / 2)$ for $\lambda=2$ and $\lambda=8$. Dashed, solid, dashed-dotted, and dotted curves correspond to $N=5,6,7$, and 8 , respectively. Units are such that $l_{0}=1$. 
shell formation in real space. Such a spatial structure is clear evidence for Wigner molecule behavior. The classical shell filling sequence has been computed recently. For $N<6$, the electrons arrange on a ring, but the sixth electron then goes into the center (shell filling 1-5). Furthermore, electrons 7 and 8 enter the outer ring again. These predictions are in accordance with our data. Clear indications of a spatial shell structure at $N \geq 6$ can be observed even for $\lambda=4$, albeit quantum fluctuations tend to wash them out. For $\lambda \gtrsim 4$, the charge densities are basically insensitive to $S$. This is characteristic for a classical Wigner crystal, where the Pauli principle and spin-dependent properties are of secondary importance. Our numerical results for the spin density in this regime simply follow the corresponding charge density according to $s_{z}(r) \simeq(S / N) \rho(r)$. A significant $S$-dependence of charge and spin densities is observed only for weak correlations.

\subsection{Crossover from Fermi liquid to Wigner molecule}

To study the crossover from weak to strong correlations, we employ the 'spin sensitivity', normalized to unity for $r_{s}=0$,

$$
\xi_{N}\left(r_{s}\right) \propto \sum_{S, S^{\prime}} \int_{0}^{\infty} d y y\left|g_{S}(y)-g_{S^{\prime}}(y)\right| .
$$

The correlation function $g_{S}(r)$ in Eq.(9) is a very sensitive measure of Fermi statistics, in particular revealing the spin-dependent correlation hole. As interactions tend to wash out the Fermi surface, the quantity (10) is largest for a Fermi gas, $r_{s}=0$. Since for $r_{s} \rightarrow \infty, g_{S}(r)$ becomes completely spin-independent, $\xi_{N}\left(r_{s}\right)$ decays from unity at $r_{s}=0$ down to zero as $r_{s} \rightarrow \infty$. The functional dependence of this decay provides insight about the crossover phenomenon under study.

Figure 2 reveals that the function $\xi_{N}\left(r_{s}\right)$ becomes remarkably universal and depends only very weakly on $N$. Its decay defines a crossover scale $r_{c}$, where an exponential fit for small $r_{s}$ yields $r_{c} \approx 4$. For $r_{s}>4$, the data can be fitted by

$$
\xi\left(r_{s}\right) \sim \exp \left(-\sqrt{r_{s} / r_{c}^{\prime}}\right),
$$

where $r_{c}^{\prime} \approx 1.2$. Remarkably, this is precisely the behavior expected from a semiclassical WKB estimate for a Wigner molecule. 3 The crossover value $r_{c} \approx 4$ is also consistent with the onset of spatial shell structures in the density, and with the spin-dependent ground state energies expected for a Wigner molecule. Therefore the crossover from weak to strong correlations is characterized by the surprisingly small value $r_{c} \approx 4$, instead of $r_{c} \approx 37$ found for the bulk 2D electron gas. 22 The stabilization of the Wigner molecule can be ascribed to the confinement potential. In the thermodynamic limit, $\omega_{0} \rightarrow 0$ with $r_{s}$ fixed, plasmons govern the low-energy physics, and hence the bulk value $r_{c} \approx 37$ becomes relevant for very large $N$. For GaAs based quantum dots, we estimate that for $N \lesssim 10^{4}$, the value $r_{c} \approx 4$ is valid. Remarkably, very recent experiments on vertical quantum dots 13 have found evidence for an even smaller crossover scale $r_{c} \approx 1.8$. The experimental study was carried out in a magnetic field, and the dot contained several impurities. Since both effects tend to stabilize a Wigner crystallized phase, our theoretical prediction and 


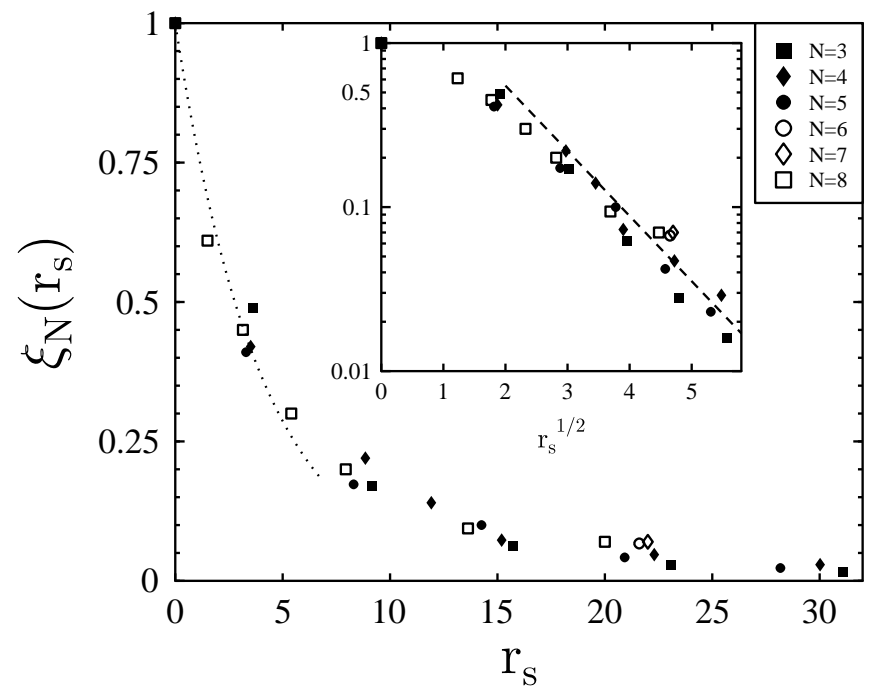

Figure 2: Numerical results for $\xi_{N}\left(r_{s}\right)$. Statistical errors are of the order of the symbol size. The dotted curve, given by $\exp \left(-r_{s} / r_{c}\right)$ with $r_{c}=4$, is a guide to the eye only. The inset shows the same data on a semi-logarithmic scale as a function of $\sqrt{r_{s}}$. The dashed line is given by Eq. (11).

the experimental observation appear to be consistent with each other, especially since we are concerned with a rather smooth crossover phenomenon.

\subsection{Spin-dependent energies}

MLB results for the energy at different parameter sets $\{N, S, \lambda\}$ are listed in Table 1. For given $N$ and $\lambda$, if the ground state is (partially) spin-polarized with spin $S$, the simulations should yield the same energies for all $S^{\prime}<S$. Within the accuracy of the calculation, this consistency check is indeed fulfilled. For strong correlations, $r_{s}>r_{c}$, the spin-dependent energy levels differ substantially from a single-particle orbital picture. In particular, the ground-state spin $S$ can change and the relative energy of higher-spin states becomes much smaller than $\hbar \omega_{0}$.

For $N=3$ electrons, as $r_{s}$ is increased, a transition occurs from $S=1 / 2$ to $S=3 / 2$ at an interaction strength $\lambda \approx 5$ corresponding to $r_{s} \approx 8$. For $N=4$, we encounter a Hund's rule case with a small- $r_{s}$ ground state characterized by $S=1$. ¿From our data, this standard Hund's rule covers the full range of $r_{s}$. A similar situation arises for $N=5$, where the ground state is characterized by $S=1 / 2$ for all $r_{s}$. Turning to $N=6$, while one has filled orbitals and hence a zero-spin ground state for weak correlations, for $\lambda=8$ we find a $S=1$ ground state. A similar transition from a $S=1 / 2$ state for weak correlations to a partially spin-polarized $S=5 / 2$ state is found for $N=7$. Finally, for $N=8$, as expected from Hund's rule, a $S=1$ ground state is observed for small $r_{s}$. However, for $\lambda \gtrsim 4$, corresponding 
Table 1: MLB data for the energy for various $\{N, S, \lambda\}$ parameter sets. Bracketed numbers denote statistical errors.

\begin{tabular}{|llll||llll|}
\hline$N$ & $S$ & $\lambda$ & $E / \hbar \omega_{0}$ & $N$ & $S$ & $\lambda$ & $E / \hbar \omega_{0}$ \\
\hline 3 & $3 / 2$ & 2 & $8.37(1)$ & 5 & $5 / 2$ & 8 & $42.86(4)$ \\
3 & $1 / 2$ & 2 & $8.16(3)$ & 5 & $3 / 2$ & 8 & $42.82(2)$ \\
3 & $3 / 2$ & 4 & $11.05(1)$ & 5 & $1 / 2$ & 8 & $42.77(4)$ \\
3 & $1 / 2$ & 4 & $11.05(2)$ & 5 & $5 / 2$ & 10 & $48.79(2)$ \\
3 & $3 / 2$ & 6 & $13.43(1)$ & 5 & $3 / 2$ & 10 & $48.78(3)$ \\
3 & $3 / 2$ & 8 & $15.59(1)$ & 5 & $1 / 2$ & 10 & $48.76(2)$ \\
3 & $3 / 2$ & 10 & $17.60(1)$ & 6 & 3 & 8 & $60.42(2)$ \\
4 & 2 & 2 & $14.30(5)$ & 6 & 1 & 8 & $60.37(2)$ \\
4 & 1 & 2 & $13.78(6)$ & 7 & $7 / 2$ & 8 & $80.59(4)$ \\
4 & 2 & 4 & $19.42(1)$ & 7 & $5 / 2$ & 8 & $80.45(4)$ \\
4 & 1 & 4 & $19.15(4)$ & 8 & 4 & 2 & $48.3(2)$ \\
4 & 2 & 6 & $23.790(12)$ & 8 & 3 & 2 & $47.4(3)$ \\
4 & 1 & 6 & $23.62(2)$ & 8 & 2 & 2 & $46.9(3)$ \\
4 & 2 & 8 & $27.823(11)$ & 8 & 1 & 2 & $46.5(2)$ \\
4 & 1 & 8 & $27.72(1)$ & 8 & 4 & 4 & $69.2(1)$ \\
4 & 2 & 10 & $31.538(12)$ & 8 & 3 & 4 & $68.5(2)$ \\
4 & 1 & 10 & $31.48(2)$ & 8 & 2 & 4 & $68.3(2)$ \\
5 & $5 / 2$ & 2 & $21.29(6)$ & 8 & 4 & 6 & $86.92(6)$ \\
5 & $3 / 2$ & 2 & $20.71(8)$ & 8 & 3 & 6 & $86.82(5)$ \\
5 & $1 / 2$ & 2 & $20.30(8)$ & 8 & 2 & 6 & $86.74(4)$ \\
5 & $5 / 2$ & 4 & $29.22(7)$ & 8 & 4 & 8 & $103.26(5)$ \\
5 & $3 / 2$ & 4 & $29.15(6)$ & 8 & 3 & 8 & $103.19(4)$ \\
5 & $1 / 2$ & 4 & $29.09(6)$ & 8 & 2 & 8 & $103.08(4)$ \\
5 & $5 / 2$ & 6 & $36.44(3)$ & & & & \\
5 & $3 / 2$ & 6 & $36.35(4)$ & & & & \\
5 & $1 / 2$ & 6 & $36.26(4)$ & & & & \\
\hline
\end{tabular}

to $r_{s} \gtrsim 10$, the ground state spin changes to $S=2$, implying a different 'strongcoupling' Hund's rule.

Let us finally address the issue of 'magic numbers'. For small $r_{s}$, the filling of orbital shells and Hund's rule arguments predict that certain $N$ are exceptionally stable. Results for the energy per electron, $E_{N} / N$, in the spin-polarized state $S=N / 2$, are shown in Figure 3. Notably, there are no obvious cusps or breaks in the $N$-dependence of the energy. Our $\lambda=2$ data in Fig. 3 suggest that an explanation of the experimentally observed magic numbers 14 has to involve spin and/or magnetic field effects. Remarkably, the absence of pronounced cusps in $E_{N} / N$ for strong correlations $(\lambda=8)$ is in accordance with the classical analysis. 8 Therefore magic numbers seem to play only a minor role in the Wigner molecule phase.

\section{Acknowledgments}

We acknowledge collaborations and discussions with Ray Ashoori, Hermann Grabert, Wolfgang Häusler, Boris Reusch, and Viktor Sverdlov. This research has been supported by the SFB 276 of the Deutsche Forschungsgemeinschaft (Bonn), by the National Science Foundation under grants CHE-9528121, CHE-9970766 and PHY94-07194, by the Sloan Foundation, and by the Dreyfus Foundation. 


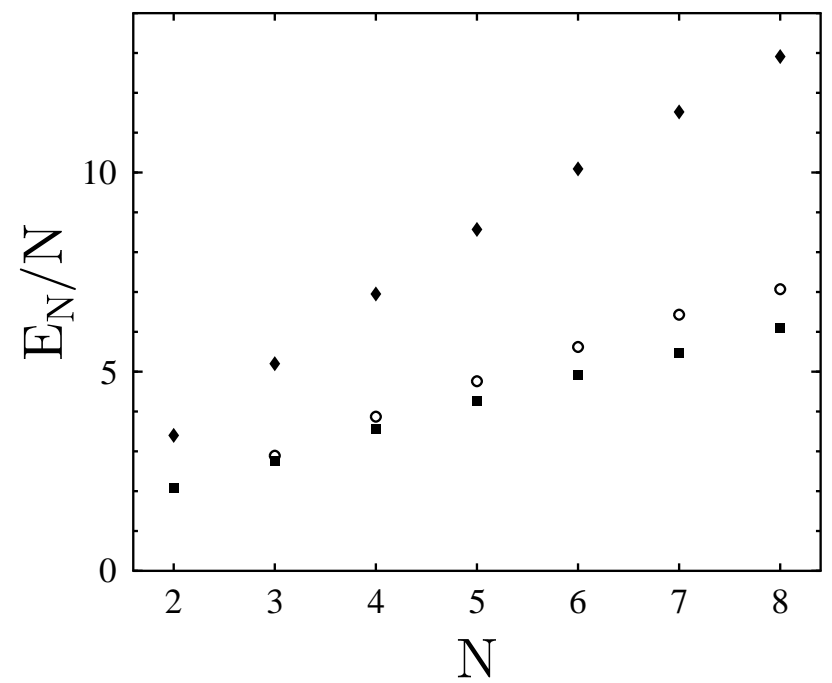

Figure 3: Energy per electron, $E_{N} / N$, for $S=N / 2$ and $\hbar \beta \omega_{0}=6$, in units of $\hbar \omega_{0}$, for $\lambda=2$ (squares) and $\lambda=8$ (diamonds). Statistical errors are smaller than the symbol size. Open circles are $T=0$ fixed-node QMC results 11 for $\lambda=2$.

\section{References}

1. E.Y. Loh Jr., J. Gubernatis, R.T. Scalettar, S.R. White, D.J. Scalapino, and R.L. Sugar, Phys. Rev. B 41, 9301 (1990).

2. C.H. Mak, R. Egger, and H. Weber-Gottschick, Phys. Rev. Lett. 81, 4533 (1998).

3. R. Egger, W. Häusler, C.H. Mak, and H. Grabert, Phys. Rev. Lett. 82, 3320 (1999); 83, 462(E) (1999).

4. C.H. Mak and R. Egger, J. Chem. Phys. 110, 12 (1999).

5. R. Egger and C.H. Mak, Phys. Rev. E (submitted).

6. C.H. Mak and R. Egger, in: New Methods in Computational Quantum Mechanics, Adv. Chem. Phys. 93, 39 (1996).

7. R.C. Ashoori, Nature 379, 413 (1996).

8. F. Bolton and U. Rössler, Superlatt. Microstruct. 13, 139 (1993); V.M. Bedanov and F.M. Peeters, Phys. Rev. B 49, 2667 (1994).

9. M. Koskinen et al., Phys. Rev. Lett. 79, 1389 (1997).

10. C. Yannouleas and U. Landman, Phys. Rev. Lett. 82, 5325 (1999).

11. F. Bolton, Phys. Rev. Lett. 73, 158 (1994).

12. B. Tanatar and D.M. Ceperley, Phys. Rev. B 39, 5005 (1989).

13. N.B. Zhitenev et al., Science 285, 715 (1999).

14. S. Tarucha et al., Phys. Rev. Lett. 77, 3613 (1996). 